\title{
Concept of Automated Machine using Mealy
}

\author{
Ritika Kalihari \\ RCET,Bhilai \\ Chhattisgarh, India
}

\author{
Toran Verma \\ RCET,Bhilai \\ Chhattisgarh,India
}

\author{
Alka Jaiswal \\ RCET,Bhilai \\ Chhattisgarh, India
}

\begin{abstract}
In recent lifestyles human require rapid processing with best quality which provides easiest lifestyle. Now-a-days, automated machines can be found everywhere in our everyday life. For example, automated machine can be found at the train stations selling train tickets. In the schools and offices automated machine provides drinks and snacks which is providing facilities to human being. And sales of goods and services via automated machine are growing strongly in India provides easiest lifestyle. Automated machine modeling is the crucial part in developing proposed model as this reduces the human resources and has been modeled using. This dissertation describes a model of an automated machine totally based on Mealy concept of FSM. The proposed model will increase the efficiency of automated machine and will also lead to cost reduction, saving time and ease of usage. The first phase involves selection of items to be purchased by consumer. In next phase, the machine generates the respective total amount required to be paid. At last the vendee enters the amount to get their products dispensed and the extra amount will be returned back by machine.
\end{abstract}

Keywords Automated machine, FSM, Mealy, Product dispensed, and Vendee.

\section{INTRODUCTION}

The first commercial coin operated machine was introduced in London and England used for selling post cards [11]. Sometimes automated Machines are used to dispense various products when money is inserted into it, in the absence of salesperson. The automated machines are more accessible and practical than the convention purchasing method. In banks as ATM machine is the best example of automated machine.

\subsection{Operation of Vending Machine [10]}

- When user presses the button the machine will tell the user that the product is available or not, for that it will display a message
- When users select the product and quantity of product, it will automatically calculate the net amount required to be paid/insert.

- User will have to now insert the specified amount for purchasing the product. Then machine will verify the amount provided.

- If extra amount is provided then it will check for change to return. If it available then it will return the change and will dispense the product. Otherwise it will cancel the product and return the amount inserted. 


\subsection{FSM CONCEPT [9],[10]}

A finite state machine (FSM) is a digital sequential circuit that consists on number of pre-defined states that are controlled by one or more inputs. The finite state machines remain stable until the inputs changes. There are two types of finite state machines: Synchronous and Asynchronous FSMs. Synchronous FSMs have a clock input and are also called Mealy machines, while asynchronous FSMs are without clock input and are called Moore machines[12]. Since, this machine is based on Mealy concept where the output is dependent on input and the present state. Only additional logic will be required simply to encode and decode the state [3] for both type of machines.

\section{Mealy Machine}

- In a Mealy machine, the outputs are a function of the present state and the value of the inputs as shown in Figure

- Accordingly, the outputs may change asynchronously in response to any change in the inputs [2].

- Mealy Machine also accepts NULL STATE.

- In Mealy Machine for $\mathbf{n}$ input there is $\mathbf{n}+\mathbf{1}$ output.

- Mealy Machine is used for Transition.

- Technically Mealy Machine can be defined as

$\mathbf{z}(\mathbf{t})=$ output $[x(t) \cdot q(t)]$

where

$$
\begin{aligned}
& x(t) \text { is input } \\
& q(t) \text { is output }
\end{aligned}
$$

- Accordingly, the outputs may change synchronously in response to any change in the inputs.

- A Moore machine can be regarded as a restricted type of finite state transducer.

- For Moore machine, output is valid after state transition.

- In Moore Machine do not accept NULL STATE for $\mathbf{n}$ input there is $\mathbf{n}$ output.

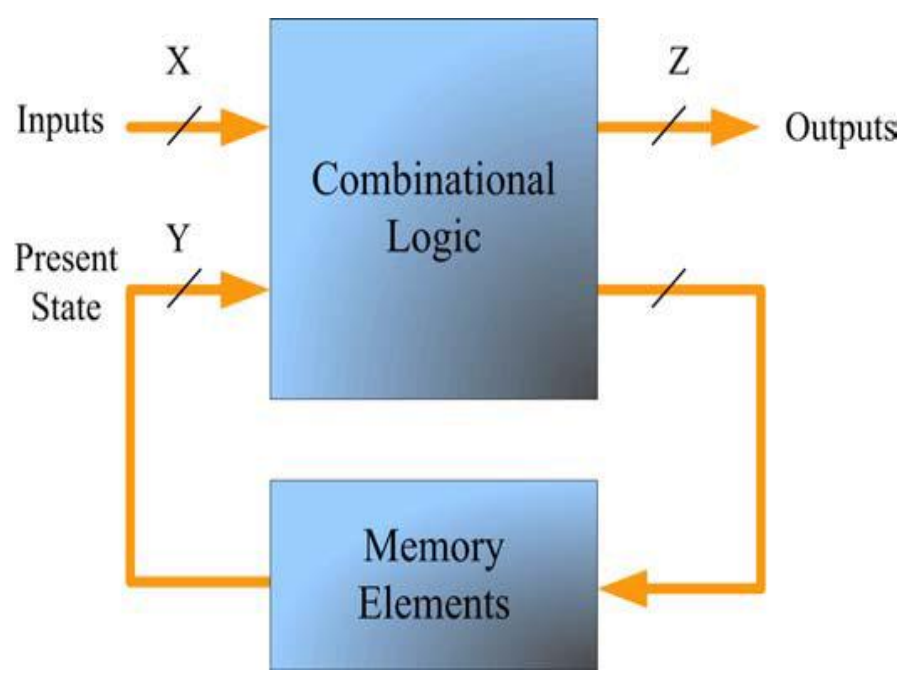

Fig. 1.1 General Diagram of Mealy machine

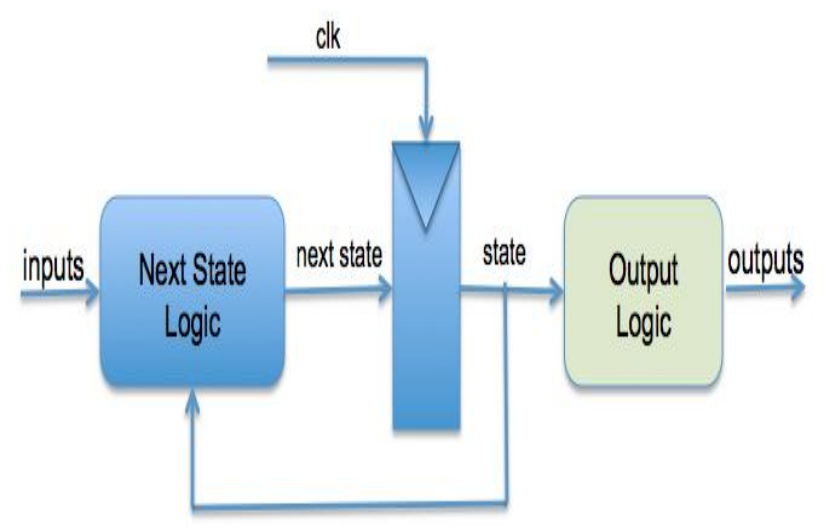

Fig. 1.2 General Diagram of Moore machine

\section{II . Moore Machine}

- In Moore machine the output values are determined only by its present states [4].

\section{LITERATURE REVIEW}

A number of researches have been carried out for designing the automated machine. Some of the works previously done are: 
2.1 A new approach is proposed in April 2012 to design an FSM based Vending Machine with auto-billing features in Vending Machine with Auto-Billing Features title paper[10].

2.2 In the year 2010 a new technique with auto billing feature for delivering the train tickets providing a new techniques for retuning greater amount implementation on Spartan-3 xc3s400 is propose and applied by Train Operating Companies (TOCs) in Train Ticketing System Using Verilog HDL [11].

2.3 This approach in 2011 is an efficient algorithm for implementation of vending machine on FPGA board is used. Because FPGA based vending machine give fast response and uses less power than the microcontroller based vending machine in the paper Vending Machine using Verilog HDL [12].

2.4 This technique shows the relationship between finite state machines and VHDL/Verilog code in Finite State Machine and VHDL Coding Techniques in year May 27-29, 2010 [13].

\section{PROPOSED METHODOLOGY}

A user friendly method is designed for any automated machine where the hardware gets reduced and also it is cost effective and time saving and convenient from the perspective of the owner and as well as the consumers. As in this the whole algorithm can be explained in one process.

In this machine model, the output depends on the present state as well as on the input as the concept is based on Mealy machine where output is dependent on present and input state.

This project is divided into 3 modules i.e.

$\begin{array}{ll}\text { 1. } & \text { Selection Module } \\ \text { 2. } & \text { Insertion Module } \\ \text { 3. } & \text { Delivery Module }\end{array}$

3.1 Selection Module: - Vendee can select the product and specify the quantity of product. The user can select more than one product at a time. For every product the entire amount will be calculated for as per the quantity selected.

www.ijcat.com
3.2 Insertion Module: - Vendee has to insert the amount for the product which he/she has selected. The amount which was inserted should not be less than the total amount i.e can be greater or equal to amount showed in the screen. So that the proper transaction can take place.

3.3 Delivery Module: - At last product will be given as output which vendee has selected according to the quantity. And rest of the amount will be returned if machine have some left amount which has to be return to the vendee.

The purpose was to improve the working rate of the automatic machine by automatically discriminating the type (structure). The designed machine can be used for many applications and we can easily enhance the number of selections. The machine is very flexible and reliable as the vendor can easily enhance the algorithm for large number of products and coins of different denominations at low cost as compared to microprocessor based vending machine. Machines accept Indian currency and give change. The machine comes with advanced features, functions and with a fully loaded payment system including Coin Change back to the user.

\section{PROPOSED METHODOLOGY}

On the basis of proposed technique a prototype of automated machine will be developed and later on simulation will be done using VHDL [5] to evaluate the performance and efficiency by considering factor like execution time of machine. For different choices of products and various coins combinations, the simulation waveforms are generated through Verilog HDL [6]. Since Verilog produces an output in a proper waveform and give fast response. And it is better choice for the implementation of mealy machine as compare to other simulation software such as Xilinx [6]. 
International Journal of Computer Applications Technology and Research

Volume 2- Issue 3, 335 - 339, 2013, ISSN: 2319-8656

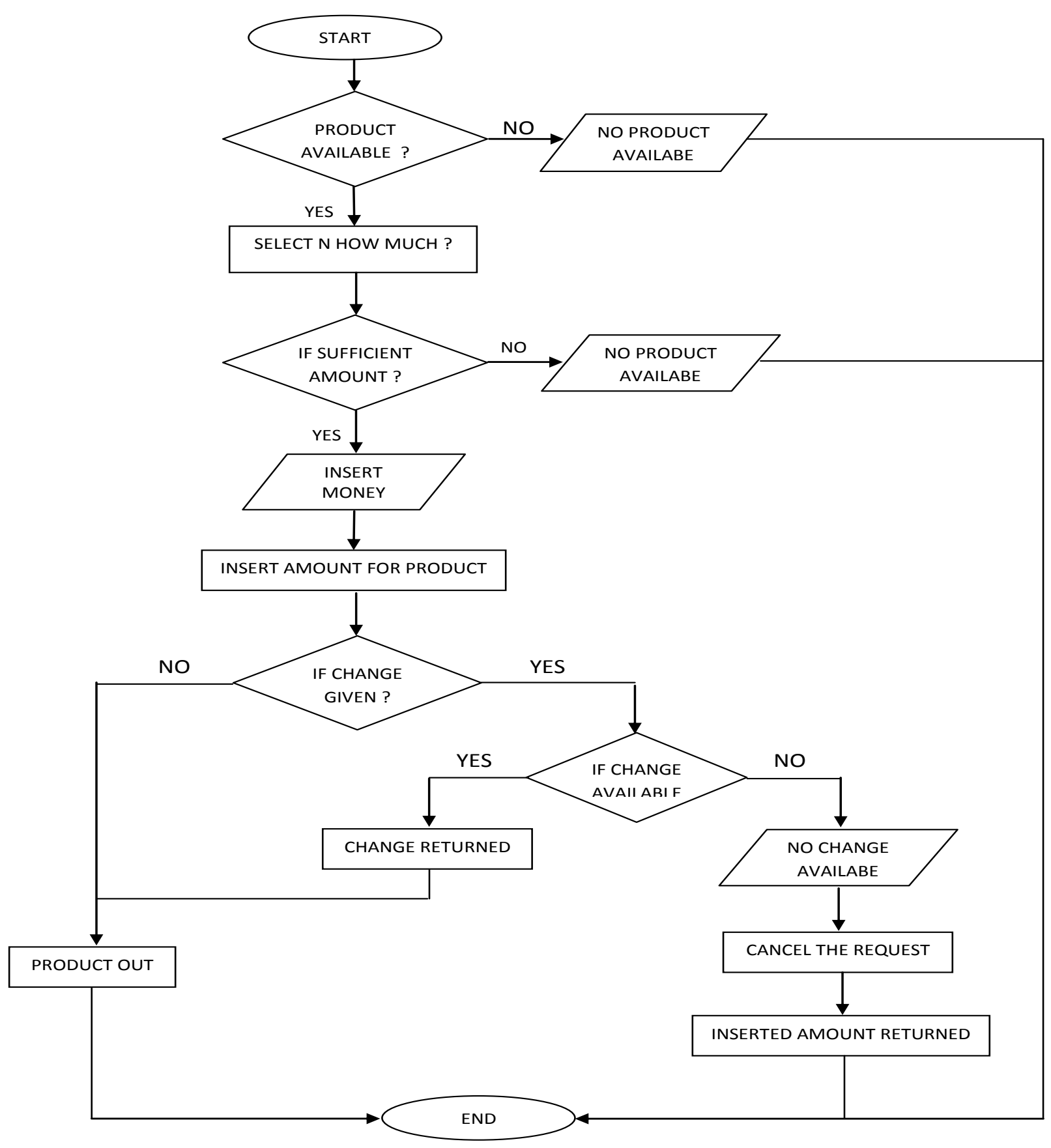

\section{CONCLUSION}

www.ijcat.com
The analysis revealed several weaknesses in the existing user interface of automated machine and a number of improvements based on the proposed design heuristics were suggested. The 


\section{International Journal of Computer Applications Technology and Research}

Volume 2- Issue 3, 335 - 339, 2013, ISSN: 2319-8656

resulting user interface would be more robust, more tolerant to user mistakes, more intuitive and efficient to use. When designing such systems it is important to apply design processes where the possible design space is adequately explored by the design team such that one can maximize the accessibility of the product accompanied by user-centric evaluations. Algorithm is very flexible and reliable as the vendor can easily enhance the algorithm for large number of products and coins of different denominations at low cost as compared to microprocessor based automated machine.

\section{REFERENCES}

[1] Fauziah Zainuddin, Norlin Mohd Ali, Roslina Mohd Sidek, Awanis Romli, Nooryati Talib \& Mohd. Izham Ibrahim (2009) "Conceptual Modeling for Simulation: Steaming frozen Food Processing in Vending.

[2] Mealy, G. H., "A method for synthesizing sequential circuits," Bell System Tech. J., Vol. 34, No. 5, pp. 1045-1079, 1955.

[3] Golson, S. , "State Machine Design Techniques for Verilog and VHDL", Synopsys Journal of High-Level Design, pp. 1-2, 1994.

[4] Moore, E. F., "Gedanken experiments on sequential machines," Automata Studies. Princeton, NJ: Princeton University Press, pp. $129-153,1956$.

[5] Volnei, A .Pedroni, "Circuit Design with VHDL", MIT Press Cambridge, Massachusetts, London, England, ISBN 0-262-16224- 5, pp. 159-186, 2004.

[6] Pong P. Chu, "FPGA Prototyping using Verilog HDL-Xilinx Spartan3 Version", John Wiley \& Sons, 2008.

[7] Biplab Roy \& Biswarup Mukherjee (2010) "Design of Coffee Vending Machine using Single Electron Devices" Proceedings of 2010 International Symposium on Electronic System Design. Pp 3843.

[8] Michael D. Ciletti, "Modeling, synthesis and Rapid prototyping with Verilog HDL"

[9] Peter Minns \& Ian Elliott, "FSM-based Digital Design using Verilog HDL", John Wiley \& Sons Ltd 2008.

[10] Ana Monga, Balwinder Singh "Finite State Machine based Vending Machine Controller with Auto-Billing Features", International Journal of VLSI design \& Communication Systems (VLSICS) Vol.3, No.2, April 2012.

[11] Muhammad Ali Qureshi, Abdul Aziz, Hafiz Faiz Rasool, "Design and Implementation of Automatic Train Ticketing System Using Verilog HDL", 2012.

[12] Muhammad Ali Qureshi, Abdul Aziz, Hafiz Faiz Rasool, Muhammad Ibrahim, Usman Ghani2 and Hasnain Abbas, "Design and Implementation of Vending Machine using Verilog HDL", 2011 2nd
International Conference on Networking and Information Technology, IPCSIT vol.17 (2011), Singapore

[13] Iuliana CHIUCHISAN, Alin Dan POTORAC, Adrian GRAUR, "Finite State Machine and VHDL coding" 